\title{
Identification of streptococcal small RNAs that are putative targets of RNase III through bioinformatics analysis of RNA sequencing data
}

\author{
Ethan C. Rath ${ }^{1}$, Stephanie Pitman ${ }^{1}$, Kyu Hong Cho ${ }^{1{ }^{*+}}$ and Yongsheng Bai ${ }^{1,2^{*+}}$ \\ From The 14th Annual MCBIOS Conference \\ Little Rock, AR, USA. 23-25 March 2017
}

\begin{abstract}
Background: Small noncoding regulatory RNAs (sRNAs) are post-transcriptional regulators, regulating mRNAs, proteins, and DNA in bacteria. One class of sRNAs, trans-acting sRNAs, are the most abundant sRNAs transcribed from the intergenic regions (IGRs) of the bacterial genome. In Streptococcus pyogenes, a common and potentially deadly pathogen, many sRNAs have been identified, but only a few have been studied. The goal of this study is to identify trans-acting sRNAs that can be substrates of RNase III. The endoribonuclease RNase III cleaves double stranded RNAs, which can be formed during the interaction between an sRNA and target mRNAs.

Results: For this study, we created an RNase III null mutant of Streptococcus pyogenes and its RNA sequencing (RNA-Seq) data were analyzed and compared to that of the wild-type. First, we developed a custom script that can detect intergenic regions of the S. pyogenes genome. A differential expression analysis with Cufflinks and Stringtie was then performed to identify the intergenic regions whose expression was influenced by the RNase III gene deletion.

Conclusion: This analysis yielded 12 differentially expressed regions with $>|2|$ fold change and $p \leq 0.05$. Using Artemis and Bamview genome viewers, these regions were visually verified leaving 6 putative sRNAs. This study not only expanded our knowledge on novel sRNAs but would also give us new insight into sRNA degradation.
\end{abstract}

Keywords: Streptococcus pyogenes, Small RNAs, RNase III, RNA sequencing, Bioinformatics

\section{Background}

S. pyogenes, also known as Group A Streptococcus (GAS), is an important human pathogen that affects 700 million people worldwide each year resulting in about 500,000 deaths due to various complications [1]. This Gram-positive bacterium can cause a wide range of both external and internal diseases. External or superficial infections include pharyngitis, impetigo, erysipelas, vaginitis, and post-partum infections [2, 3]. Although detrimental, these diseases are not typically fatal. However, some can progress to necrotizing fasciitis and

\footnotetext{
* Correspondence: KyuHong.Cho@indstate.edu; Yongsheng.Bai@indstate.edu ${ }^{\dagger}$ Equal contributors

'Department of Biology, Indiana State University, Terre Haute, IN 47809, USA

Full list of author information is available at the end of the article
}

scarlet fever which are far more problematic [4]. Internally, GAS can cause necrotizing fasciitis, cellulitis, septic arthritis, puerperal sepsis, meningitis, abscess, osteomyelitis, endocarditis, and peritonitis, all which can involve toxic shock-like syndrome [5]. The type of resulting infection is determined by how the bacteria are contracted and by the serotype. Serotypes are determined by the sequence on the $5^{\prime}$ end of the $\mathrm{emm}$ gene, which encodes for $M$ protein [6]. However, there are many different factors beyond $M$ protein that affect GAS virulence [7]. Further understanding of the mechanisms behind $S$. pyogenes infections could lead to new ways of treating this pathogen. Likewise, more knowledge of the pathogenesis of this pathogen could play a 
role in comparatively studying similar mechanisms in other pathogens.

The most ubiquitous post-transcriptional regulator across all bacteria is the small non-coding regulatory RNA (sRNA) [8]. These sRNAs are incredibly important in translational regulation by controlling mRNA activity [9]. Although less common, some sRNAs can also affect DNA and proteins [10-12]. These sRNAs come in two major types: cis-acting sRNAs (cis-sRNAs) and transacting sRNAs (trans-sRNAs). The defining characteristic of cis-RNA is that it is transcribed from the same region as its target (antisense cis-sRNA) or even within the same mRNA as its potential target (sense cis-sRNA) [13]. Cis-sRNA typically targets either the gene (or genes) with which it is transcribed or the gene(s) opposite to it (Fig. 1a) [13]. Cis-sRNAs have a much more limited scope of targets than trans-sRNAs, which could be any in the RNA transcriptome. As such, this paper is focused on the discovery of trans-sRNA.

Trans-sRNAs employ many different modes of action to regulate RNA expression [14]. They can repress
mRNA translation, enhance mRNA translation, increase degradation of mRNA, or block degradation. Some sRNA can function in multiple manners, depending on their targets. These mechanisms have been described in detail and are usually dependent on the sRNA binding site on the target mRNA. Unlike cis-sRNAs, trans-sRNAs are transcribed from intergenic regions (IGRs) throughout the genome (Fig. 1b), making these sRNA easier to detect both computationally and through visual analysis [15]. The development of sRNA identification-related bioinformatics tools has allowed for the prediction of many novel sRNAs in a wide range of bacteria, including $S$. pyogenes [13]. Some sRNAs are involved in regulating virulence of pathogens, and to date, three transsRNAs have been identified as regulators of virulence factors in S. pyogenes, PelRNA, RivX, and FasX [8, 16-18]. By studying basic sRNA degradation, our research has the potential to eventually be applied to the research of the degradation of virulence factor-regulating sRNAs as well.

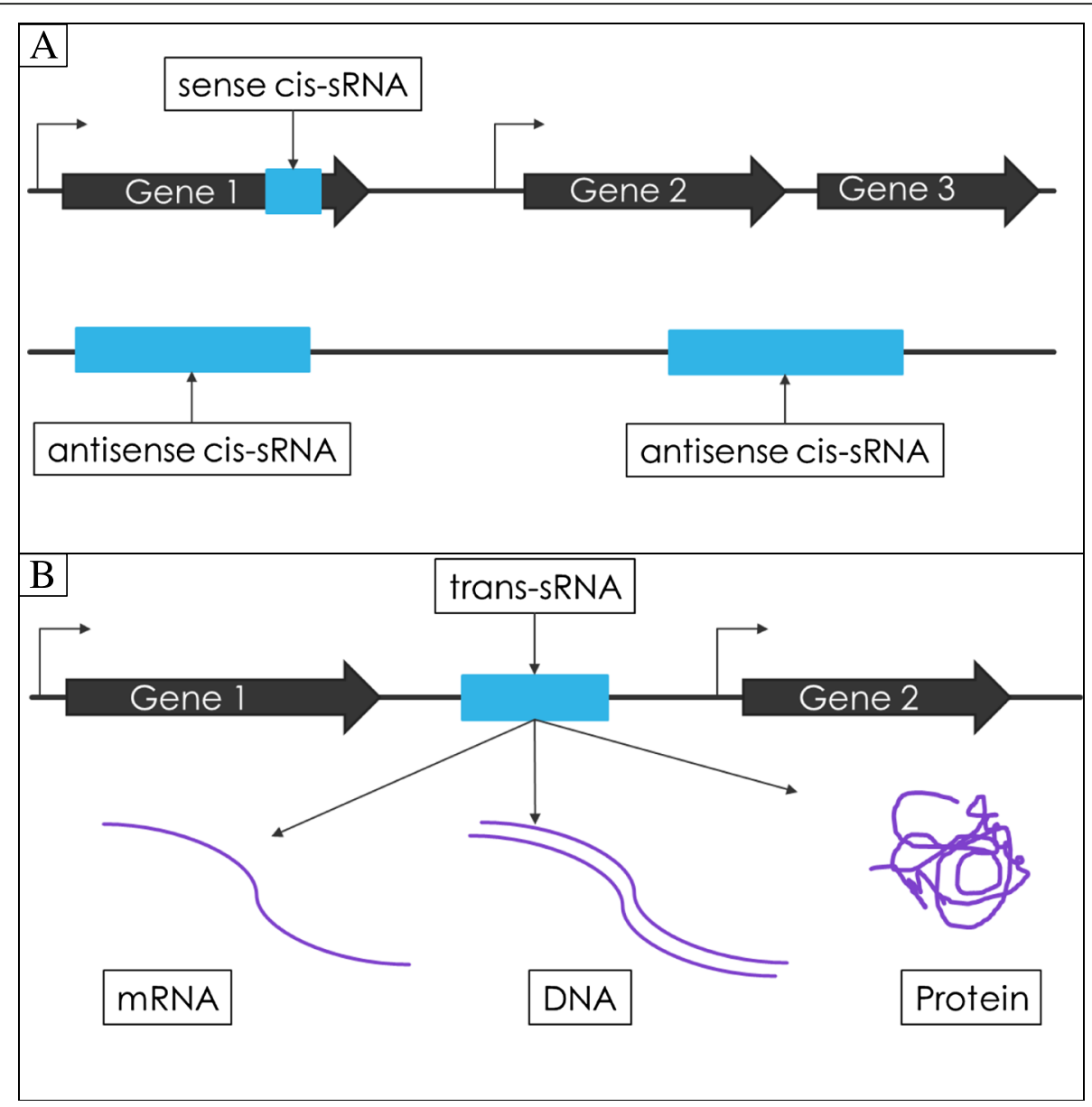

Fig. 1 a Types of cis-sRNAs and their gene locations in the chromosome (b) The gene location of trans-sRNA and its targets. Thick black arrows represent theoretical genes and their directions, blue boxes denote theoretical locations of sRNA genes, and purple items represent targets of a trans-sRNA 
Currently, the degradation of sRNAs is not well known. It is thought that the degradation of sRNA is reliant on various ribonucleases similar to the process of mRNA degradation. Ribonucleases are enzymes that assist in the breakdown of RNA in cells [19]. The endoribonuclease RNase III whose substrates are doublestranded RNAs has shown great promise as a potential post-transcriptional regulator of many sRNAs [20, 21].

The RNA sequencing (RNA-seq) data processing has been simplified to an easy to use open source pipeline that has been widely used for alignment and observation of RNA-seq data. This process relies on many different algorithms that can yield multiple types of results, from simple alignments to complex differential expression analyses. This pipeline starts with Bowtie2 as a base which creates an index of the genomic file [22]. The index created here is used for alignment of the short reads by the Burrow-Wheeler transformation algorithm (BWA) based RNA alignment software TopHat [23]. After alignment, the BAM (or SAM) files are then passed to Cufflinks [24, 25]. Cufflinks processes the alignments into an assembled form. All assemblies are sent to Cuffmerge for further analysis [24, 25]. Finally, Cuffdiff compares the expression of each transcript through read depth and identifies any differentially expressed transcripts [24, 25]. We also applied a second pipeline named as the RSEM/EBSeq pipeline [26-28] for differential expression cross analysis. This pipeline requires complete annotation of unannotated regions, provided by StringTie [26]. This annotation is then used to align, assemble, calculate expression, and merge these values into a matrix using RSEM [27]. Lastly, differential expression is performed using EBSeq [28].

The goal of this study was to identify differentially expressed IGRs potentially affected by the endoribonuclease RNase III in $S$. pyogenes and then determine whether they are sRNAs. First, a bioinformatics approach was developed by employing two different pipelines (Figs. 2, 3) to analyze the RNA sequencing data of the wild type strain, HSC5, and an RNase III null mutant for differential expression. The IGRs reflecting $>2$ fold-difference were analyzed visually to determine any potential sRNAs.

\section{Methods \\ Streptococcus pyogenes growth condition}

$S$. pyogenes HSC5 was used for all experiments and strain construction. HSC5 is a non-mucoid M14 serotype lab strain [29], and has recently been sequenced [30].Todd Hewitt media (BBL) with $0.2 \%$ yeast extract (THY media) was used to cultivate $S$. pyogenes. For growth in liquid media, S. pyogenes was cultured at $37^{\circ}$ $\mathrm{C}$ in sealed tubes without shaking. To produce solid media, Bacto agar was added to a final concentration of $1.4 \%$ (wt/vol). S. pyogenes grown on plates (solid media) was incubated in anoxic conditions using the Gas Pak EZ anaerobe containment system (Catalogue no. 260678, BBL). Escherichia coli Top10 (Invitrogen) was used for plasmid construction. E. coli was cultured in Luria-Bertani broth (LB) at $37{ }^{\circ} \mathrm{C}$ with shaking. When appropriate, optimum concentration of antibiotics was added to the media.

\section{Creation of a nonpolar in-frame deletion mutant of the RNase III gene, rnc}

An in-frame deletion allele of $r n c$ was created as follows. The primers of 5outRNase3IFD-КpnI (aaaggtacccaaagagttagcgcatatgacg) and 3outRNase3IFD (cagtatctttagtctgtctttcttgagc) were used to amplify a $2.02 \mathrm{~kb}$ DNA fragment including $r n c$. This amplified fragment was digested and inserted between $K p n \mathrm{I}$ and $\mathrm{XbaI}$ restriction sites in the multiple cloning site of pCRII (Invitrogen). The $K p n I$ restriction site is located in the primer sequence of 5outRNase3IFD-KpnI, which is underlined, and the XbaI site is located near the 3 ' end of the PCR-amplified product. The resulting plasmid was then used as a template in an 'inside-out' PCR reaction with the primers of $5 \mathrm{inRNa}$ se3IFD-XmaI (aaacccgggattagtgagaaaggacctgccc) and 3inRNase3IFD-XmaI (aacccgggctctgaaataatcaattgtagaacagcg). Restriction of this fragment with XmaI followed by subsequent re-ligation resulted in a nonpolar inframe deletion that replaces DNA sequence encoding Y61 - V181

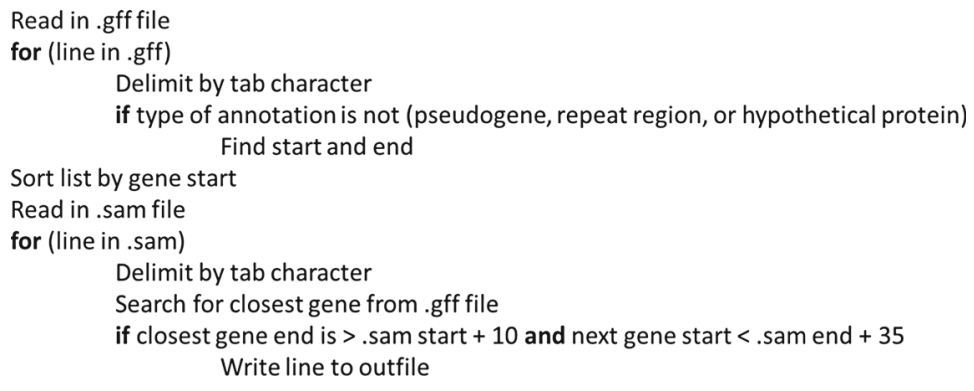

Fig. 2 Pseudocode for intergenic region detection script 


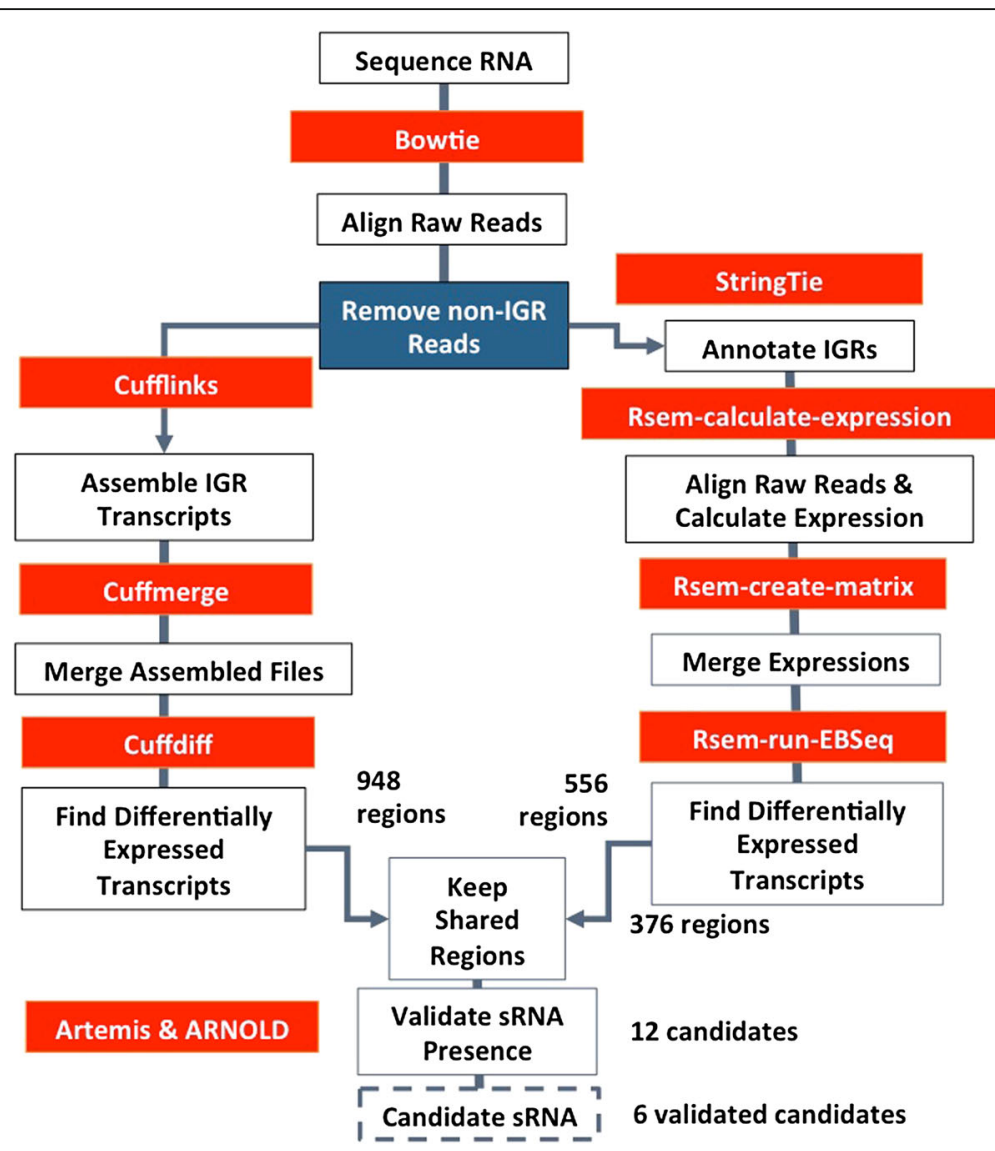

Fig. 3 Pipeline for RNA-seq analysis for intergenic region detection. Red boxes denote software used while the solid blue box is software developed in house. This pipeline details two separate pipelines for analyzing RNA-seq data focusing on intergenic regions with measureable expression. Both pipelines were used to create a final prospective list, also denoted in this workflow

of RNase III with the sequence of cccggg encoding PG. The in-frame deletion allele of $r n c$ was then inserted between the BamHI and XbaI restriction site in the $S$. pyogenes-E. coli shuttle vector, pJRS233. The generated plasmid, pJRS233::rnc-IFD, was used to replace the wild type $r n c$ with the in-frame-deleted $r n c$ by a method that employs the temperature sensitivity of the pJRS233 replication origin [31].

\section{RNA extraction from S. pyogenes}

The wild type (HSC5) and the RNase III mutant ( $\triangle$ RNase III) were grown in THY media to the exponential phase (OD600 of 0.4-0.5). Then, total RNA was extracted using the combination of the miRNeasy kit (Qiagen) and the FastPrep beadbeater (MP biomedicals). An S. pyogenes cell pellet from $10 \mathrm{ml}$ culture was resuspended in $700 \mathrm{ml}$ of the Qiazol lysis reagent (Qiagen) and transferred to a Lyse Matrix B blue cap tube (MP biomedicals). Cells were then lysed by the beadbeater, FastPrep 24 (MP biomedicals) at the speed of 6.0 for $40 \mathrm{~s}$ twice. The remaining procedure for RNA extraction followed the manufacturer's protocol of the miRNeasy kit. During RNA purification, RNase-Free DNase (Qiagen) was treated on column to remove residual DNA. The A260/A280 ratio of the extracted RNA was measured with Eppendorf BioSpectrometer ${ }^{\circ}$ to determine the RNA concentration and purity (accepted if $>1.8$ ). The extracted RNA was mixed with 1 ul of RNasin (Promega Recombinant RNasin Ribonuclease Inhibitor, $40 \mathrm{u} / \mathrm{ml}$ ), and treated with the RNAstable kit for safe transport.Next-generation sequencing, RNA-Seq.

The extracted RNA samples were submitted to Macrogen Corporation (Rockvill, MD, USA) for RNA-Seq assays. The quantity, integrity and purity of total RNA were assessed using Ribogreen (Life technologies, cat\# R11490) and Agilent Bioanalyzer 2100. RNA was subjected to rRNA depletion using the RiboZero MetaBacteria kit (Epicentre Biotechnologies, Madison, WI USA, catalog \# MRZMB126) and cDNA was generated from the rRNA depleted RNA using the NEBNext mRNA Sample Prep kit (New England Biolabs, Ipswich, MA USA, catalog\# E6110). cDNA was profiled using Agilent Bioanalyzer, and subjected to Illumina library preparation using NEB Next reagents (New England 
Biolabs, Ipswich, MA USA, catalog\# E6040). The quality and quantity and the size distribution of the Illumina libraries were determined using an Agilent Bioanalyzer 2100. The libraries were then submitted for Illumina HiSeq2000 sequencing according to the standard operation. Paired-end 90 or 100 nucleotide (nt) reads were generated.

\section{Alignment of RNA-seq data}

To detect sRNAs expressed from the RNA-seq data, raw sequencing data was aligned using TopHat [23] with a Bowtie2 index file [22] to the HSC5 genome.fasta (GCF_000422045.1_ASM42204v1_genomic.fna). TopHat produced four BAM files for each condition (2 for each replicate). These were converted into SAM files using samtools [32]. A custom Python script was written to report the aligned reads that appeared in the IGRs of the HSC5 genome. This script takes in a SAM file and an annotated genome file (in .gff format) and identifies any reads that fall within an IGR. An IGR was defined as a location that was $10 \mathrm{bp}$ downstream from the end of a known gene and 35 bp upstream from the next downstream gene. Any reads found within these regions were then written into a new SAM file (Fig. 2).

\section{Intergenic region detection}

Once the reads that aligned to IGRs were detected and isolated, each SAM file was loaded into the differential expression pipeline provided by the Cufflinks package [22-25] (Fig. 2). Each SAM file was run through Cufflinks and then merged through Cuffmerge. These files were then used for a final run through Cuffdiff [22-25]. The final differential expression from the Cufflinks pipeline gave 938 potential regions.

\section{Alternate differential expression}

In order to confirm the regions detected by Cufflinks, a secondary pipeline for differential expression analysis using RSEM was used [26-28]. RSEM requires an annotated genome in order to perform a differential expression analysis, therefore our detected regions had to be annotated. The SAM files produced by our novel script were processed through the Stringtie software to annotate the IGRs that were detected by our program [26]. The headers of these files were removed and they were concatenated into a singular .gff file. This .gff along with the raw sequence files were used for rsem-calculateexpression. The transcript files produced from the first step were then combined into an expression matrix using rsem-generate-data-matrix. Finally, this matrix was run through rsem-run-ebseq [27]. EBSeq differential expression analysis yielded 556 differentially expressed IGRs [28].

\section{Final candidate choice}

The results of both Cufflinks differential expression and EBseq were compared to find similar regions using a custom Python script. A total of 376 regions were detected in common by both pipelines and selected for further analysis. Any statistically significant differentially expressed regions with at least a 2 -fold change and a $p$-value $\leq 0.05$ were used for further analysis.

\section{Visual confirmation}

Using the genome viewer tools Artemis and Bamview, the statistically significant differentially expressed IGRs were analyzed for visual confirmation as sRNAs [33]. Respective regions in WT and RNase III mutant RNAseq data were compared.

\section{ARNold confirmation}

Most sRNAs have rho-independent terminators at their 3 ' ends. As such, prediction software was used to determine if these terminators were present in any of the current candidates. Using IGVs regions of interest, we compiled the sequences of each candidate IGR into a multi-fasta file. This file was uploaded into ARNold and run using default settings [34-36]. The results were then used to further classify the candidates.

\section{Results and Discussion}

Creation and confirmation of an RNase III gene inframe deletion mutant

The interaction between an sRNA and its mRNA target forms a double strand RNA structure that can be the substrate of RNase III. Therefore, RNase III could be an enzyme at least in part responsible for the degradation or processing of some sRNAs. To test this possibility, a deletion of the $r n c$ gene encoding RNase III was performed, and the expression of putative sRNAs of the mutant was compared to that of the wild type.

There are several strategies to inactivate genes in Streptococcus: allelic replacement, directed insertional inactivation, and in-frame deletion. The first two methods are relatively easy to carry out. However, the mutated gene could generate an undesired expression pattern of the downstream genes in the same operon. Sequence analysis showed that immediately downstream of $r n c$, there was a gene with the same direction, encoding the putative chromosomal partition protein SMC. Since the distance between both genes is short, they might form an operon. For this reason, the in-frame deletion approach was used to delete rnc.

In the in-frame deletion method, the gene of interest is inserted into a vector whose replication is dependent on temperature. Then, a large central portion of the gene is deleted in-frame, which preserves the reading frame of the message. After inserting a DNA containing 
$r n c$ and its flanking regions into the E. coli $-S$. pyogene shuttle vector pJRS233, we deleted 372 bp that represents $54 \%$ of the $r n c$ sequence through an inverse PCR technique. We confirmed through DNA sequencing that the construct contained the deletion and still maintained the reading frame. Later, the construct was transferred to the wild type HSC5 strain, and necessary steps were performed to obtain the mutant. PCR was performed to confirm the in-frame deletion in the chromosome (Fig. 4).

\section{Intergenic region detection and differential expression}

Regulation of multiple components of the bacterial cell can be performed by the expression of sRNAs. The trans-acting sRNAs, in particular, are often effective at regulating multiple different transcripts as opposed to the typically limited scope of cis-acting sRNAs. Since trans-acting sRNAs are transcribed from IGRs, detection of these sRNAs relies on the accurate identification of IGRs. In order to be classified as a putative sRNA, the region had to adhere to the following characteristics: the differentially expressed region of interest is between two genes, and a significant difference in read depth between the region of interest and the flanking genes exists, so the differential expression does not appear to be a result

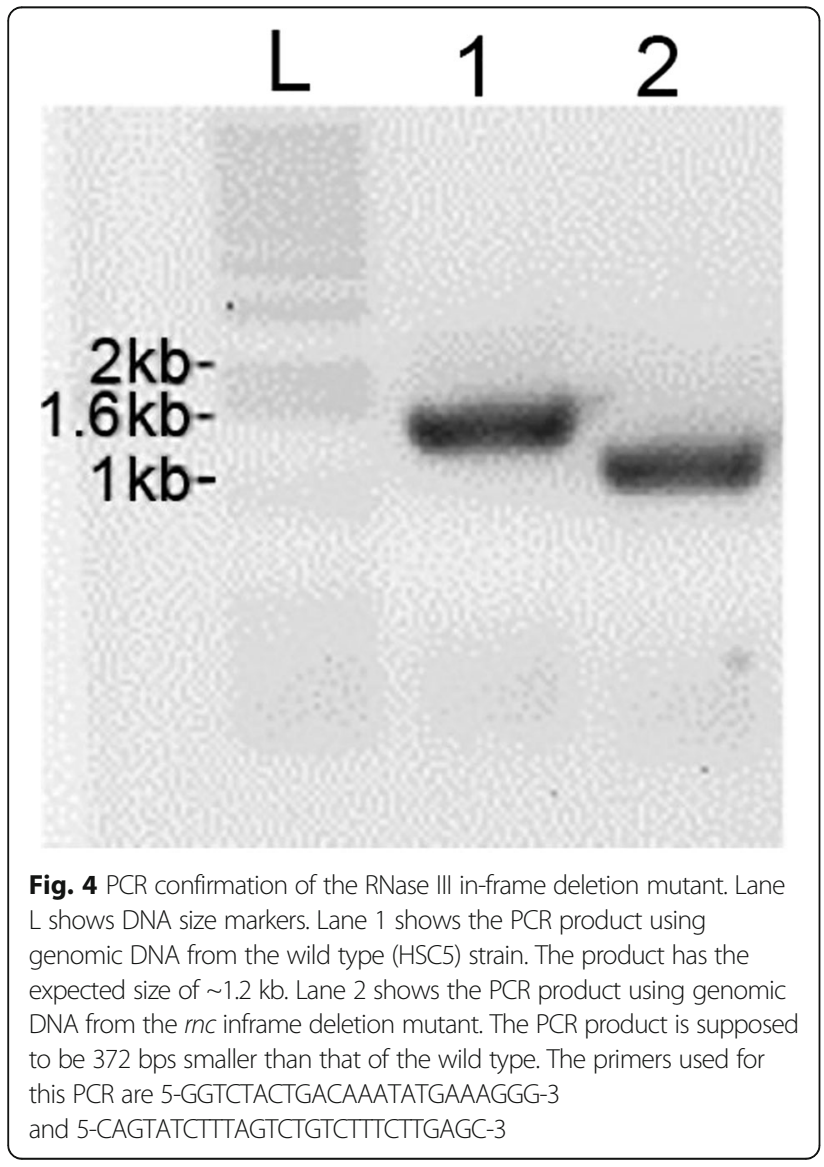

of flanking gene transcription. If both flanking genes faced the same direction, the read depth was then evaluated. When flanking genes pointed in the same direction and showed similar read depth including the IGR, the region was labeled as an operon structure and eliminated from sRNA candidates. The regions with read depths that were not entirely equivalent to the nearby genes were labeled as possible sRNAs.

For this detection, the entire transcriptome of wildtype HSC5 (WT) and the RNase III null mutant ( $\triangle$ RNase III) were sequenced. Using the raw RNA-seq data, two differential expression pipelines Cufflinks and RSEM/EBSeq were executed, adding a step after Tophat alignment (Fig. 3). This additional step included a novel script written in Python that identified the RNA transcripts that were transcribed from IGRs (Fig. 2). Cufflinks identified 948 differentially expressed regions, while RSEM/EBSeq discovered 556 regions. The results of both pipelines were combined to find the IGRs that were detected by both programs. Within these IGRs, 376 were identified as having some manner of differential expression between WT and $\Delta$ RNase III. In order to select the best potential regions for further testing, those regions with a $p$-value $\leq 0.05$ and an absolute fold change $>2$ were identified, leaving us with 12 potential regions (Table 1). These regions were then confirmed through visual analysis and rhoindependent terminator predictions.

\section{Confirmation of detected regions}

In order to ascertain the validity of the potential regions identified above, we turned to an in silico visual confirmation using the Artemis software provided by the Sanger Institute. Visual confirmation detected regions involved in operon structure or regions that have low enough read support to be determined to be false positives. We proved the final decisions on the 12 identified regions, with 6 of these $(\sim 50 \%)$ being confirmed as either likely or highly likely sRNA (Table 1 ). For added clarity, three examples of these visual confirmations are shown in Fig. 4. Operon structures were determined by gene direction and by uniformity of read depth as depicted in Fig. 5b.

For further confirmation, we also looked for predicted rho-independent terminators within the regions that were detected by our software pipeline. The ARNold software uses two different methods for prediction. The top predictions are shown in Table 1. The terminators detected were then confirmed through manual checks. If the predicted terminator was at a location to allow for the termination of putative sRNAs, rather than the flanking gene regions, it was utilized for the ranking of sRNA potential. These predictions were combined with the visual confirmation. Any region with both positive 
Table 1 Detected IGRs with potential for sRNA expression and their confirmation

\begin{tabular}{|c|c|c|c|c|c|}
\hline TSS\# $^{\mathrm{a}}$ & Fold Change ( $\Delta$ RNase III/HSC5) & $p$-Value & Visual Confirmation using Bamview & Predicted Rho-independent Terminator & sRNA Potential \\
\hline 59 & -2.81 & $5.00 \mathrm{E}-05$ & Negative & Yes & Unlikely \\
\hline 72 & 4.42 & $5.00 \mathrm{E}-05$ & Negative & No & Highly Unlikely \\
\hline 241 & 2.77 & 0.0216 & Negative & No & Highly Unlikely \\
\hline 231 & 2.48 & 0.00095 & Negative & Yes & Likely \\
\hline 627 & 2.22 & 0.00465 & Negative & No & Highly Unlikely \\
\hline 53 & 2.26 & 0.0436 & Negative & Yes & Unlikely \\
\hline 333 & 2.37 & 0.00135 & Positive & No & Likely \\
\hline 516 & 2.24 & 0.02875 & Positive & No & Likely \\
\hline 332 & 2.29 & $5.00 \mathrm{E}-05$ & Positive & Yes & Highly Likely \\
\hline 795 & 2.33 & 0.02665 & Positive & No & Likely \\
\hline 181 & 2.05 & 0.0059 & Positive & No & Likely \\
\hline 520 & 2.10 & 0.0405 & Positive & Yes & Highly Likely \\
\hline
\end{tabular}

${ }^{\mathrm{a} A n}$ arbitrary numeric identifier provided for unannotated region by Cufflinks

visual validation and a manually validated terminator was considered a highly likely sRNA. If visual validation was positive but no terminator was detected then the region was considered a likely sRNA. A region with only a putative terminator was considered unlikely to be an sRNA while those without any supporting evidence were considered highly unlikely. This analysis left 6 out of the 12 regions to be considered likely sRNAs or better. The detailed region information for these potential sRNAs is given in Table 2.

The RNase III family of endoribonucleases has been well studied across all organisms, from bacterial RNase III to the Drosha/Dicer family in eukaryotes [37]. Bacterial RNase III typically contains two major regions: the endonuclease domain followed by a double-stranded RNA (dsRNA) binding domain [38]. RNase III is able

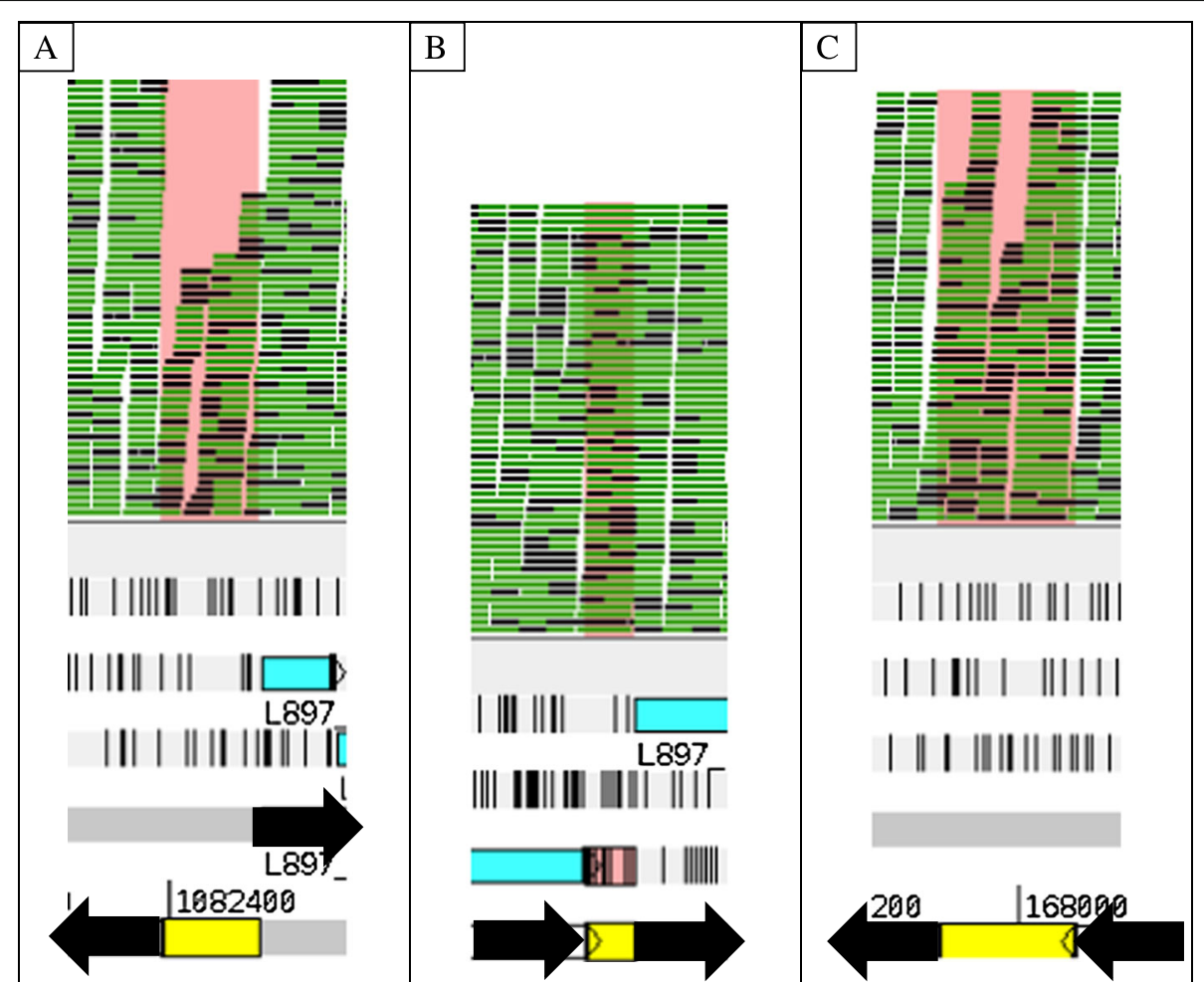

Fig. 5 Examples of visual confirmation of sRNAs using Artemis Genome Viewer. Pink highlighted areas represent regions detected by our software and black arrows were added to show flanking gene direction. (a) An IGR encoding a putative sRNA, (b) An IGR in a predicted operon structure, and (c) A IGR encoding a putative sRNA even though the flanking genes are in the same direction 
Table 2 The information of IGRs encoding a putative sRNAs that are differentially expressed in $\Delta$ RNase III compared to the wild type

\begin{tabular}{|c|c|c|c|}
\hline TSS \# & Left Gene (Direction) & Right Gene (Direction) & Sequence $^{a}$ \\
\hline TSS333 & L897_03295 (Ł) & L897_03295 $(\rightarrow)$ & $\begin{array}{l}\text { ATCTCAGATTAAATTATACCAAAAATGTGAAGCTAA } \\
\text { TGCTTGTTAAGTCAAATTAGTAGGATIIITATCAGA } \\
\text { TITGTTATAATAAAAACTATGAATAAACTCTATATTGATT } \\
\text { CTITGTCGAAAAGAAGCTGACAGCAGGGGT } \\
\text { ACAATTATTAGATGA }\end{array}$ \\
\hline TSS516 & L897_04905 $(\rightarrow)$ & L897_04905 (Ł) & $\begin{array}{l}\text { GACTTTCTITAAACTATGACACACTATAGTTIA } \\
\text { AAAGAAAGTIIITCAGTGTTCATAGTAAAT } \\
\text { AAAAAAACCGTCTTCCATCAAATAGAAGCGGTTIAT } \\
\text { CAAATTAACACCAAACCTTAATGCTGTAAGAACCAA } \\
\text { GATATAACATCTITCCAAAAATAAATAAATAACTGT } \\
\text { AGCCGAT }\end{array}$ \\
\hline TSS332 & L897_03290 $(\rightarrow)$ & L897_03290 (Ł) & $\begin{array}{l}\text { ACCTAGAAAATAACTITTATTACCTATAGAAAGTTA } \\
\text { TAAAGAAACAAAAATGAAGGAGACGATGGACGT } \\
\text { CTICTITTATTATACTCAATATAATAAAAAGAAGTTा } \\
\text { CCCATGTITATTACCAGTAATGTGGGATATTIAGATG } \\
\text { GTAGCAAGAAGTTTATAGTTGATTTGTTCTITAGGT } \\
\text { CTAATAAGCATATITGATTACTGATAAACTTGAATA } \\
\text { TCGCCAATAAAAAAGAAGCAAAATTATTATTTTGCTTCTA } \\
\text { GTCAGATCATTAAATTGATTATTCTCTATITTGGTGTTA } \\
\text { TCCTCTITAGTTCAGAAGTTGCCGCGTCAGCTTCTACGGGAT }\end{array}$ \\
\hline TSS795 & L897_07905 (Ł) & L897_07905 $(\rightarrow)$ & $\begin{array}{l}\text { CCACACTAAAATGAGATTAGTTAATCATGTTAAGTTAT } \\
\text { TAAAAACTTCGGTIITATGAAGTCAAGTIITAGAGAG } \\
\text { TIITAGACCATCTITACGATACCTITGCTITAACCTCTIIT } \\
\text { ATGGTATCATATITATATAAAGAAAAGGAGAAAAATATG } \\
\text { TCCGCCAAGAAAACTIIITTGCAAGTAATTAAAGTACCTTA } \\
\text { GATTAAAAAAGAACATGG }\end{array}$ \\
\hline TSS181 & L897_01895 $(\rightarrow)$ & L897_01895 (Ł) & $\begin{array}{l}\text { AAGCTCTCGTGTCCCCTATCACATGCATAGGATCAGT } \\
\text { GCACTCGACCTITCAAGACAAGCAAGCATCAGCTCTTGCTTGT } \\
\text { CTIIITTGGCCTCAAAGCCCGTAATCTGCTGCTATGCGAGG } \\
\text { CTIIITGAGCATCAGAACGTCAAAAAAAAGGACATGGAGTC } \\
\text { CTIIITGGTGATCGGTGTGAGGCCGTCACAAACTGCCCTTGA } \\
\text { AATACGCTTCTATGTGGAGCTITITTGGCCTGTGACACGTAA } \\
\text { GCTCC }\end{array}$ \\
\hline TSS520 & L897_04925 (Ł) & L897_04925 (Ł) & $\begin{array}{l}\text { CAATTTGCTTAGCAAGTATACTATATTIAAATAATAATTCAA } \\
\text { CTATAATITAAAAAAACACAAAAAAAACATTATACAGCTAT } \\
\text { AAAGCTTAATATAATAGGATITATGTATACAATTATTAAC } \\
\text { AGCATCTATTCAAGATCGCCTACTTCATCAGGTTGGTATGA } \\
\text { CTAAGTIITAACTTATCTICCCCCCTIIITGTITAGAA } \\
\text { GATAAAAGAATITCTTGATITGCACACAAAAAACCGCCCTCA } \\
\text { ACTAAGAGAGCGGTTGGTTTTTATTTAAGGAGACAGTGACT }\end{array}$ \\
\hline
\end{tabular}

${ }_{\text {aitalics denote predicted terminator regions }}$

to regulate gene expression through RNA degradation or binding to target RNAs. RNase III binding helps to stabilize its targets, which can then affect the translation of downstream genes [39-42]. Cleavage of dsRNA by RNase III has been shown to be a major post-transcriptional regulation [43]. Transcripts targeted by RNase III are cleaved into pieces that are average 10-18 bp in length. For a description of the complex mechanics of this process, see Gan et al. but put simply, dsRNA is detected and bound to the dsRNA binding domain, which then cleaved via hydrolysis in the catalytic site of the RNase III homodimer [37, 44]. It is highly plausible that dsRNA formed between an sRNA and its target mRNA could be a substrate of RNase III [45], and our study discovered the putative sRNAs that can be
RNase III substrates. The targets and regulatory mechanisms of the putative sRNAs identified from this study could be further studied in the future. A better understanding of sRNA degradation could be used to help study various sRNAs, including those potentially involved in virulence regulation.

\section{Conclusions}

Through the development of an automatic step to add to the typical RNA-seq processing pipeline that detects and isolates the reads that align to IGRs, our study has shown that it is effective in finding potential sRNAs. Through visual validations, we were able to estimate that our methods had a recovery rate of $50 \%$ for finding potential sRNAs for the studied samples in $S$. pyogenes. 


\section{Abbreviations}

cDNA: Complementary DNA; DNA: Deoxyribonucleic Acid; dsRNA: Double Stranded Ribonucleic Acid; GAS: Group A Streptococcus; GFF: Genome File Format; IGR: Intergenic Regions; mRNA: messenger Ribonucleic Acid; PCR: Polymerase Chain Reaction; RNA: Ribonucleic Acid; RNA-seq: Ribonucleic Acid Sequencing; S. pyogenes: Streptococcus pyogenes; SAM: Sequence Alignment Map; sRNA: small Noncoding-Ribonucleic Acid; WT: Wild-type; $\triangle$ RNase III: Ribonuclease III null Streptococcus pyogenes mutant

\section{Acknowledgements}

We would like to acknowledge the help of Dr. Dewey for his valuable assistance in this research.

\section{Funding}

Funding for this project and publication costs was provided by Indiana State University Grants URC and COMPETE to Yongsheng Bai. This study was also supported in part by National Institutes of Health Grant 7R15 GM101603-02 to Kyu Hong Cho. The funding body was not involved with the design of the study, analysis, and interpretation of data or in the writing of the manuscript.

\section{Availability of data and materials}

All customized programs developed in this study are publically available and the data used are available through the corresponding author.

\section{About this supplement}

This article has been published as part of BMC Bioinformatics Volume 18 Supplement 14, 2017: Proceedings of the 14th Annual MCBIOS conference. The full contents of the supplement are available online at https:// bmcbioinformatics.biomedcentral.com/articles/supplements/volume-18supplement-14.

\section{Authors' contributions}

ER prepared and wrote the manuscript, performed all bioinformatics analysis, and wrote all code for this project. SP assisted in manuscript writing, performed visual validation, and provided background information. $\mathrm{KHC}$ designed experimental procedures, created the mutant strain, performed RNA-sequencing, wrote the corresponding parts and revised the manuscript. YB conceived the study, guided the bioinformatics analysis, revised and finalized the manuscript. All authors critically read manuscript drafts and approved the final version of the manuscript.

\section{Ethics approval and consent to participate}

Not applicable.

\section{Consent for publication}

All authors consent for the publication of this research.

\section{Competing interests}

The authors declare that they have no competing interests.

\section{Publisher's Note}

Springer Nature remains neutral with regard to jurisdictional claims in published maps and institutional affiliations.

\section{Author details}

${ }^{1}$ Department of Biology, Indiana State University, Terre Haute, IN 47809, USA. ${ }^{2}$ The Center for Genomic Advocacy, Indiana State University, Terre Haute, IN 47809, USA.

\section{Published: 28 December 2017}

\section{References}

1. Carapetis JR, Steer AC, Mulholland EK, Weber M. The global burden of group a streptococcal diseases. Lancet Infect Dis. 2005;5(11):685-94.

2. Wessels MR. Clinical practice. Streptococcal pharyngitis. N Engl J Med. 2011; 364(7):648-55.

3. Choby BA. Diagnosis and treatment of streptococcal pharyngitis. Am Fam Physician. 2009:79(5):383-90.

4. Celestin R, Brown J, Kihiczak G, Schwartz RA. Erysipelas: a common potentially dangerous infection. Acta Dermatovenerol Alp Pannonica Adriat. 2007;16(3):123-7.
5. Henningham A, Barnett TC, Maamary PG, Walker MJ. Pathogenesis of group a streptococcal infections. Discov Med. 2012;13(72):329-42.

6. Bessen DE. Tissue tropisms in group a streptococcus: what virulence factors distinguish pharyngitis from impetigo strains? Curr Opin Infect Dis. 2016; 29(3):295-303.

7. Sarkar P, Sumby P. Regulatory gene mutation: a driving force behind group a streptococcus strain- and serotype-specific variation. Mol Microbiol. 2017; 103(4):576-89.

8. Brantl S, Bruckner R. Small regulatory RNAs from low-GC gram-positive bacteria. RNA Biol. 2014;11(5):443-56.

9. Masse $E$, Gottesman S. A small RNA regulates the expression of genes involved in iron metabolism in Escherichia Coli. Proc Natl Acad Sci U S A. 2002:99(7):4620-5.

10. Babitzke P, RomeoT. CsrB sRNA family: sequestration of RNA-binding regulatory proteins. Curr Opin Microbiol. 2007:10:156-63.

11. Gimpel M, Brantl S. Dual-function small regulatory RNAs in bacteria. Mo Microbiol. 2017:103(3):387-97.

12. Gottesman S. Micros for microbes: non-coding regulatory RNAs in bacteria. Trends Genet. 2005:21(7):399-404.

13. Cho KH, Kim JH. Cis-encoded non-coding antisense RNAs in streptococci and other low GC gram (+) bacterial pathogens. Front Genet. 2015;6:110.

14. Bobrovskyy M, Vanderpool CK. Regulation of bacterial metabolism by small RNAs using diverse mechanisms. Annu Rev Genet. 2013;47:209-32.

15. Pitman $\mathrm{S}, \mathrm{Cho} \mathrm{KH}$. The mechanisms of virulence regulation by small noncoding RNAs in low GC gram-positive pathogens. Int J Mol Sci. 2015; 16(12):29797-814

16. Roberts SA, Scott JR. RivR and the small RNA RivX: the missing links between the CovR regulatory cascade and the Mga regulon. Mol Microbiol. 2007;66(6):1506-22.

17. Kreikemeyer B, Boyle MD, Buttaro BA, Heinemann M, Podbielski A. Group a streptococcal growth phase-associated virulence factor regulation by a novel operon (Fas) with homologies to twocomponent-type regulators requires a small RNA molecule. Mol Microbiol. 2001;39(2):392-406.

18. Ramirez-Pena E, Trevino J, Liu Z, Perez N, Sumby P. The group a streptococcus small regulatory RNA FasX enhances streptokinase activity by increasing the stability of the ska mRNA transcript. Mol Microbiol. 2010;78(6):1332-47.

19. Belasco JG. All things must pass: contrasts and commonalities in eukaryotic and bacterial mRNA decay. Nat Rev Mol Cell Biol. 2010;11(7):467-78.

20. Viegas SC, Silva IJ, Saramago M, Domingues S, Arraiano CM. Regulation of the small regulatory RNA MicA by ribonuclease III: a target-dependent pathway. Nucleic Acids Res. 2011;39(7):2918-30.

21. Lioliou E, Sharma CM, Caldelari I, Helfer AC, Fechter P, Vandenesch F, Vogel J, Romby P. Global regulatory functions of the Staphylococcus Aureus endoribonuclease III in gene expression. PLoS Genet. 2012; 8(6):e1002782.

22. Langmead B, Trapnell C, Pop M, Salzberg SL. Ultrafast and memory-efficient alignment of short DNA sequences to the human genome. Genome Biol. 2009;10(3):R25.

23. Trapnell C, Pachter $L$, Salzberg SL. TopHat: discovering splice junctions with RNA-Seq. Bioinformatics. 2009;25(9):1105-11.

24. Trapnell C, Hendrickson DG, Sauvageau M, Goff L, Rinn JL, Pachter L. Differential analysis of gene regulation at transcript resolution with RNA-seq Nat Biotechnol. 2013:31(1):46-53.

25. Trapnell C, Roberts A, Goff L, Pertea G, Kim D, Kelley DR, Pimentel H, Salzberg SL, Rinn JL, Pachter L. Differential gene and transcript expression analysis of RNA-seq experiments with TopHat and cufflinks. Nat Protoc. 2012;7(3):562-78.

26. Pertea M, Pertea GM, Antonescu CM, Chang TC, Mendell JT, Salzberg SL. StringTie enables improved reconstruction of a transcriptome from RNA-seq reads. Nat Biotechnol. 2015:33(3):290-5

27. Li B, Dewey CN. RSEM: accurate transcript quantification from RNA-Seq data with or without a reference genome. BMC Bioinform. 2011:12:323.

28. Leng N, Dawson JA, Thomson JA, Ruotti V, Rissman Al, Smits BM, Haag JD, Gould MN, Stewart RM, Kendziorski C. EBSeq: an empirical Bayes hierarchical model for inference in RNA-seq experiments. Bioinformatics. 2013;29(8): 1035-43.

29. Hanski E, Caparon M. Protein F, a fibronectin-binding protein, is an adhesin of the group a streptococcus streptococcus pyogenes. Proc Natl Acad Sci U S A. 1992;89(13):6172-6. 
30. Port GC, Paluscio E, Caparon MG. Complete genome sequence of emm type 14 streptococcus pyogenes strain HSC5. Genome Announc. 2013;1 (4):612-13.

31. Cho KH, Kang SO. Streptococcus pyogenes c-di-AMP phosphodiesterase, GdpP, influences SpeB processing and virulence. PLoS One. 2013;8(7): e69425.

32. Li H, Handsaker B, Wysoker A, Fennell T, Ruan J, Homer N, Marth G, Abecasis G, Durbin R. Genome project data processing S: the sequence alignment/ map format and SAMtools. Bioinformatics. 2009;25(16):2078-9.

33. Zhang J, Zheng YG. SAM/SAH analogs as versatile tools for SAM-dependent Methyltransferases. ACS Chem Biol. 2016;11(3):583-97.

34. Lesnik EA, Sampath R, Levene HB, Henderson TJ, McNeil JA, Ecker DJ. Prediction of rho-independent transcriptional terminators in Escherichia Coli. Nucleic Acids Res. 2001;29(17):3583-94.

35. Gautheret D, Lambert A. Direct RNA motif definition and identification from multiple sequence alignments using secondary structure profiles. J Mol Biol. 2001;313(5):1003-11.

36. Macke TJ, Ecker DJ, Gutell RR, Gautheret D, Case DA, Sampath R. RNAMotif, an RNA secondary structure definition and search algorithm. Nucleic Acids Res. 2001;29(22):4724-35.

37. Gan J, Tropea JE, Austin BP, Court DL, Waugh DS, Ji X. Structural insight into the mechanism of double-stranded RNA processing by ribonuclease III. Cell. 2006;124(2):355-66.

38. Lamontagne B, Larose S, Boulanger J, Elela SA. The RNase III family: a conserved structure and expanding functions in eukaryotic dsRNA metabolism. Curr Issues Mol Biol. 2001;3(4):71-8.

39. Oppenheim AB, Kornitzer D, Altuvia S, Court DL. Posttranscriptional contro of the lysogenic pathway in bacteriophage lambda. Prog Nucleic Acid Res Mol Biol. 1993;46:37-49.

40. Calin-Jageman I, Nicholson AW. RNA structure-dependent uncoupling of substrate recognition and cleavage by Escherichia Coli ribonuclease III. Nucleic Acids Res. 2003;31(9):2381-92.

41. Dasgupta S, Fernandez L, Kameyama L, Inada T, Nakamura Y, Pappas A, Court DL. Genetic uncoupling of the dsRNA-binding and RNA cleavage activities of the Escherichia Coli endoribonuclease RNase III-the effect of dsRNA binding on gene expression. Mol Microbiol. 1998;28(3):629-40.

42. Guarneros G, Kameyama L, Orozco L, Velazquez F. Retroregulation of an intlacZ gene fusion in a plasmid system. Gene. 1988;72(1-2):129-30.

43. Bardwell JC, Regnier P, Chen SM, Nakamura Y, Grunberg-Manago M, Court DL. Autoregulation of RNase III operon by mRNA processing. EMBO J. 1989; 8(11):3401-7.

44. Gan J, Shaw G, Tropea JE, Waugh DS, Court DL, Ji X. A stepwise model for double-stranded RNA processing by ribonuclease III. Mol Microbiol. 2008; 67(1):143-54.

45. Saramago M, Barria C, Dos Santos RF, Silva IJ, Pobre V, Domingues S, Andrade JM, Viegas SC, Arraiano CM. The role of RNases in the regulation of small RNAs. Curr Opin Microbiol. 2014;18:105-15.

\section{Submit your next manuscript to BioMed Central and we will help you at every step:}

- We accept pre-submission inquiries

- Our selector tool helps you to find the most relevant journal

- We provide round the clock customer support

- Convenient online submission

- Thorough peer review

- Inclusion in PubMed and all major indexing services

- Maximum visibility for your research

Submit your manuscript at www.biomedcentral.com/submit

) Biomed Central 